\title{
Search for agents causing atypical pneumonia in HIV-positive patients by inhibitor-controlled PCR assays
}

\author{
B. Tarp*, J.S. Jensen**, L. Østergaard*, P.L. Andersen*
}

\begin{abstract}
Search for agents causing atypical pneumonia in HIV-positive patients by inhibitor-controlled PCR assays. B. Tarp, J.S. Jensen, L. Østergaard, P.L. Andersen. (C) ERS Journals Ltd 1999.

ABSTRACT: Pneumonia is one of the most frequent complications in acquired immunodeficiency syndrome-patients with Pneumocystis carinii as the leading cause. The true prevalence of atypical agents such as Chlamydia pneumoniae, $C$. trachomatis, Legionella pneumophila and Mycoplasma pneumoniae in this population of patients is unknown as the currently used method for diagnosing these agents is measurement of antibody levels. However, this method is of limited value in human immunodeficiency virus (HIV)-positive patients who may have a compromised antibody response.

To evaluate the prevalence of Chlamydia spp., Legionella spp. and M. pneumoniae in HIV-infected patients with pulmonary disease, this retrospective study has applied inhibitor-controlled polymerase chain reaction analyses on 103 bronchoalveolar lavage (BAL) fluids representing 103 episodes of pneumonia in $83 \mathrm{HIV}$-positive patients.

$L$. pneumophila was detected in $1 \%$ of the BAL fluids and $M$. pneumoniae was found as a coexisting pathogen in $2 \%$ of the samples. Chlamydia spp. could not be detected in any of the BAL fluids. By culture and staining methods 106 other microorganisms were detected with $P$. carinii and Streptococcus pneumoniae as the most frequently occurring.

Pneumonia due to Chlamydia pneumoniae, Legionella pneumophila or Mycoplasma pneumoniae seems to be rare in Danish human immunodeficiency virus-infected patients, but might be considered as a possible cause in cases of treatment failure. Eur Respir J 1999; 13: 175-179.
\end{abstract} \author{
Denmark. \\ Correspondence: B. Tarp \\ Dept of Infectious Diseases \\ Marselisborg Hospital \\ P.P. Ørumsgade 11 \\ Aarhus University Hospital \\ DK-8000 Aarhus C \\ Denmark \\ Fax: 4589491800
}

*Dept of Infectious Diseases, Aarhus University Hospital, Aarhus C, Denmark. **Mycoplasma Laboratory, Statens Serum Institut, Artillerivej 5, Copenhagen S,

Keywords: Atypical pneumonia human immunodeficiency virus polymerase chain reaction

Received: February 281998

Accepted after revision August 141998

This work was supported by grants from the National Association Against Lung Diseases and Scandinavian Society for Antimicrobial Chemotherapy.
Pneumonia is the most frequent complication in acquired immunodeficiency syndrome (AIDS) patients, with Pneumocystis carinii as the leading cause being involved in $20-80 \%$ of cases [1-3]. The empirical antibiotic treatment in AIDS patients with pneumonia is therefore directed against $P$. carinii and consists of trimethoprim-sulphamethoxazole(TMP-SMX). Although high-doses ofTMP-SMX have been shown to be effective in human immunodeficiency virus (HIV)-positive patients infected with Legionella pneumophila [4], this treatment is, however, not optimal for other agents causing atypical pneumonia such as Chlamydia spp. and Mycoplasma pneumoniae. The recommended drugs for treatment of these micro-organisms are primarily macrolides or tetracyclines. Thus, an exact diagnosis of organisms causing atypical pneumonia is needed in order to establish the optimal antibiotic treatment in AIDS patients with pneumonia, especially since the ratio of agents causing atypical pneumonia/P. carinii pneumonia (PCP) may increase as a consequence of highly active antiretroviral therapy (HAART) which may reduce the number of PCP cases.

A diagnosis of Chlamydia spp., L. pneumophila or M. pneumoniae pneumonia cannot be obtained on a clinical or radiographical basis [5] and direct detection of the micro-organisms is associated with great difficulties [6, 7]. The antibody response is compromised in AIDS patients and measurements of antibody levels is therefore of lim- ited value $[8,9]$. Chlamydia pneumoniae has been detected in $8-10 \%$ of bronchoalveolar lavage (BAL) fluids from HIV-positive patients $[10,11]$. In BAL fluids or sputum from HIV-positive patients with pulmonary infections, $L$. pneumophila has been infrequently detected, but when it is detected it is frequently as a coexisting pathogen or nosocomial infection $[4,8,12]$. M. pneumoniae is rare in adults as well as children with HIV-infection $[13,14]$. The prevalence of atypical agents causing pneumonia in HIVinfected patients is unknown as no evaluated routine method for diagnosing these agents in HIV-positive patients currently exists.

In a retrospective study, an inhibitor-controlled polymerase chain reaction (PCR) analyses for Chlamydia spp., Legionella spp. and M. pneumoniae has been applied on consecutively obtained BAL fluids from HIV-infected patients with pneumonia in order to evaluate the prevalence of these agents.

\section{Materials and methods}

\section{Patient samples}

BAL fluids from HIV-positive patients undergoing fibreoptic bronchoscopy for suspected pneumonia between March 1991 to December 1995 at the Department of Infectious Diseases, Aarhus University Hospital, Aarhus, 
Denmark were included. One hundred and three BAL fluids representing 103 episodes of pneumonia were obtained from 83 patients. The median age of the patients was 35 yrs (range: 27-62 yrs). The median CD4 count was $47 \times 10^{6} \cdot \mathrm{L}^{-1}$ (range: $1-297$ ). Thirty-eight per cent of the patients received azidothymidine (AZT), 7\% dideoxyinosine (DDI) and the remaining 55\% did not receive any type of antivirals. Twenty patients underwent BAL more than once. The BAL was performed in accordance with standard techniques by instillation of three aliquots of $60 \mathrm{~mL}$ $0.9 \%$ sodium chloride, preheated to $37^{\circ} \mathrm{C}$, into the radiographically abnormal region of the lung. In cases with or without diffuse infiltrates, lavage was performed in the middle lobe and/or lingula. The BAL fluids were immediately stored at $-40-80^{\circ} \mathrm{C}$ until analysed.

Sample preparation for M. pneumoniae, chlamydia and legionella PCR

At the Mycoplama Laboratory, Statens Serum Institut, the samples were prepared as follows: $300 \mu \mathrm{L}$ of the BAL fluid was centrifuged at $30,000 \times g$ for $15 \mathrm{~min}$. Deoxyribonucleic acid (DNA) from the pellet was released by thorough vortexing with $300 \mu \mathrm{L} 20 \%$ (w/v) Chelex 100 slurry (BioRad, Hercules, CA, USA) [15] followed by boiling of the suspension for $10 \mathrm{~min}$.

At the Research Laboratory, Department of Infectious Diseases, Aarhus University Hospital, the samples were prepared as follows: the BAL fluid was centrifuged at $20,000 \times g$ for $30 \mathrm{~min}$. The supernatant was removed and DNA was extracted by use of the Amplicor HVC reverse transcription (RT)-PCR specimen preparation kit (Roche Diagnostics, Branchburg, NJ, USA) according to the manufacturer's instructions.

\section{General PCR conditions}

All PCR assays were used with a "Hot Start" protocol [16] performed by adding $10 \mu \mathrm{L} 1 \times$ PCR reaction buffer (withheld from the master mix) containing the appropriate amount of Taq DNA polymerase to the tubes after heating to $80^{\circ} \mathrm{C}$.

An internal processing control (IPC) consisting of a 616 base pairs (bp) phage lambda DNA flanked by the PCR primers was used in order to detect inhibition of the PCR. If inhibition was observed by lack of amplification of the IPC, smaller volumes of the pretreated sample were tested in the PCR, however, at least $5 \mu \mathrm{L}$ sample was tested, otherwise the assay was deemed to be inhibited.

Strict precautions were taken throughout all procedures in order to minimize the risk of PCR product carryover as previously described [17]. All surfaces in the PCR set-up laboratory were regularly wiped with a $4 \%$ Diversol solution containing hypochlorite [18] and exposed to ultraviolet (UV)-light between sessions to destroy contaminating DNA.

\section{Chlamydia $P C R$}

The pretreated sample $(10 \mu \mathrm{L})$ was subjected to a PCR using the primer pair $1 \mathrm{~A} / 1 \mathrm{~B}$ [19] and corresponding mixed primers matching the $16 \mathrm{~S}$ ribosomal DNA (rDNA) se- quence of other Chlamydia spp. A "touchdown" procedure [20] was used with a $1{ }^{\circ} \mathrm{C}$ decrement of the annealing temperature for the first 10 cycles. A total of 50 cycles were performed. Amplicons were visualized by staining with ethidium bromide after agarose gel electrophoresis. Amplified material was labelled during PCR with digoxigenin-11deoxyuridine triphosphate (dUTP) (Boehringer-Mannheim, Mannheim, Germany) at a molar ratio to unlabelled dUTP of 1:312. In order to lower the detection limit and to differentiate between the three chlamydial species, a liquid hybridization assay using biotinylated species specific probes was used. This assay is consistently capable of detecting $<0.2$ inclusion forming units of all three chlamydial species.

The presence of $C$. pneumoniae and $C$. trachomatis was further assessed by use of nested PCR for detection of $C$. pneumoniae and $C$. trachomatis as previously described $[21,22]$.

\section{M. pneumoniae $P C R$}

The pretreated sample $(25 \mu \mathrm{L})$ was tested with primers P1-178/P1-331 [23] deduced from the M. pneumoniae P1 adhesin gene in a 40 cycle two-step PCR. Amplicons were detected by agarose gel electrophoresis. Positive results were confirmed with a second primer pair detecting another part of the M. pneumoniae P1 gene. Both assays had a detection limit of $<5-10$ genome copies.

\section{Legionella PCR}

For the detection of Legionella spp. two PCR assays were used. A legionella genus-specific assay amplified a fragment of the legionella 16S rRNA gene [24], whereas a L. pneumophila specific sequence of the mip gene was amplified with primers PT69, PT70 and PT181 available from the EnviroAmp Legionella PCR Amplification Kit (Perkin Elmer Cetus, Foster City, CA, USA). Pretreated sample $(10 \mu \mathrm{L})$ was tested in each of the two assays. The cycling conditions were identical to those described for the chlamydia PCR. Amplicons were detected by agarose gel electrophoresis. Both PCR assays had a detection limit of $<5-10$ colony-forming units (cfu).

M. pneumoniae, chlamydia and legionella PCR was performed at the Mycoplasma Laboratory, Statens Serum Institut, Copenhagen, Denmark. Nested-PCR for detection of $C$. pneumoniae and C. trachomatis was performed at the Research Laboratory, Department of Infectious Diseases, Aarhus University Hospital, Aarhus, Denmark.

\section{Other microbiological examinations}

Cultures for bacteria inclusive of Legionella spp. and fungi in BAL fluid were performed at the Department of Clinical Microbiology, Aarhus University Hospital, Aarhus, Denmark. Cultures of M. pneumoniae and Chlamydia spp. were not performed.

The detection of $P$. carinii was performed by use of immunofluorescence or Gomori methenamine silver stain at the Laboratory of Parasitology, Aarhus University Hospital, Aarhus, Denmark and the Department of Pathology, Aarhus University Hospital, Aarhus, Denmark. 


\section{Results}

\section{Detection of Chlamydia species}

Inhibition of these methods was found in three of 103 BAL fluid samples representing 103 episodes of pneumonia. Of the 100 eligible BAL fluid samples no Chlamydia spp. could be detected by either of the three chlamydia PCR methods.

\section{Detection of Legionella species}

No samples showed inhibition in the legionella PCR. Of the 103 samples, L. pneumophila was detected in only one $(1 \%)$. This sample was obtained from a male patient with a CD4 count of $6 \times 10^{6} \cdot \mathrm{L}^{-1}$. This patient received DDI as an antiviral. At the time of admission the patient was treated with ampicillin and itraconazol due to chronic sinusitis and oral candidiasis. The patient had high fever $\left(39.9^{\circ} \mathrm{C}\right)$, cough but no clinical signs of pneumonia or radiographic infiltrates. From the BAL fluid of this patient Candida albicans and cytomegalovirus (CMV; 500 virus particles $\cdot \mathrm{mL}^{-1}$ ) were cultured. The culture of legionella was negative and $P$. carinii was not detected, but owing to the suspicion that $P$. carinii could be the aetiological agent, treatment with TMP-SMX and prednisone was introduced, resulting in some recovery. After one month, however, the patient still had fever and an infiltrate had appeared. Ceftazidime was given without effect and 7 weeks later the patient died. No autopsy was performed. The detection of L. pneumophila by PCR was not performed until after the patient had died.

\section{Detection of $\mathrm{M}$. pneumoniae}

Inhibition of the M. pneumoniae PCR was found in eight of the samples, but after testing of a lower sample volume, all samples could be evaluated. Of the 103 samples, $M$. pneumoniae was detected in two $(2 \%)$ male patients with CD4 counts of $50 \times 10^{6} \cdot \mathrm{L}^{-1}$ and $32 \times 10^{6} \cdot \mathrm{L}^{-1}$, respectively.
Both of the patients received AZT as antivirals. Before admission the first patient was treated with penicillin without any effect. The patient had fever $\left(39.6^{\circ} \mathrm{C}\right)$, cough and was slightly hypoxic and a chest radiograph showed smallspotted, bilateral infiltrates. In addition to M. pneumoniae both $P$. carinii, Staphylococcus aureus and CMV were detected in the BAL fluid. Treatment with high-dose TMPSMX was given and within 12 days the patient recovered. The other patient had had fever, cough and shortness of breath for the last three weeks prior to the admission. At the time of admission the symptoms were still present but there was no stetoscopic signs of pneumonia or radiographic infiltrates. S. pneumoniae was also isolated from the BAL fluid and treatment with roxithromycin was started and the patient recovered. Thus, M. pneumoniae was present as a coexisting pathogen in two of 103 samples. Both cases occurred in a period of no epidemics in Denmark (fig. 1).

\section{Detection of other agents}

A total of 106 organisms were detected in 103 BAL fluids. The BAL fluids were not examined for viruses except for $\mathrm{CMV}$ in a few cases. In 23 of the BAL fluids more than one organism were detected. In 35 cases (34\%) no organisms were found. The prevalence of various organisms are shown in table 1.

\section{Discussion}

C. pneumoniae has been detected by PCR and culture in $8-10 \%$ of the BAL fluids from HIV positive patients $[10,11]$. However, the authors were unable to confirm these findings, although two different $C$. pneumoniae PCR methods were used to demonstrate Chlamydia spp. in 100 BAL fluids from HIV-positive patients with signs of lower respiratory tract infection. It is difficult to explain these differences, but these studies do not support the assumption that $C$. pneumoniae should be a common cause of pneumonia in Danish HIV-positive patients or that $C$. pneumoniae should colonize the respiratory tract in these

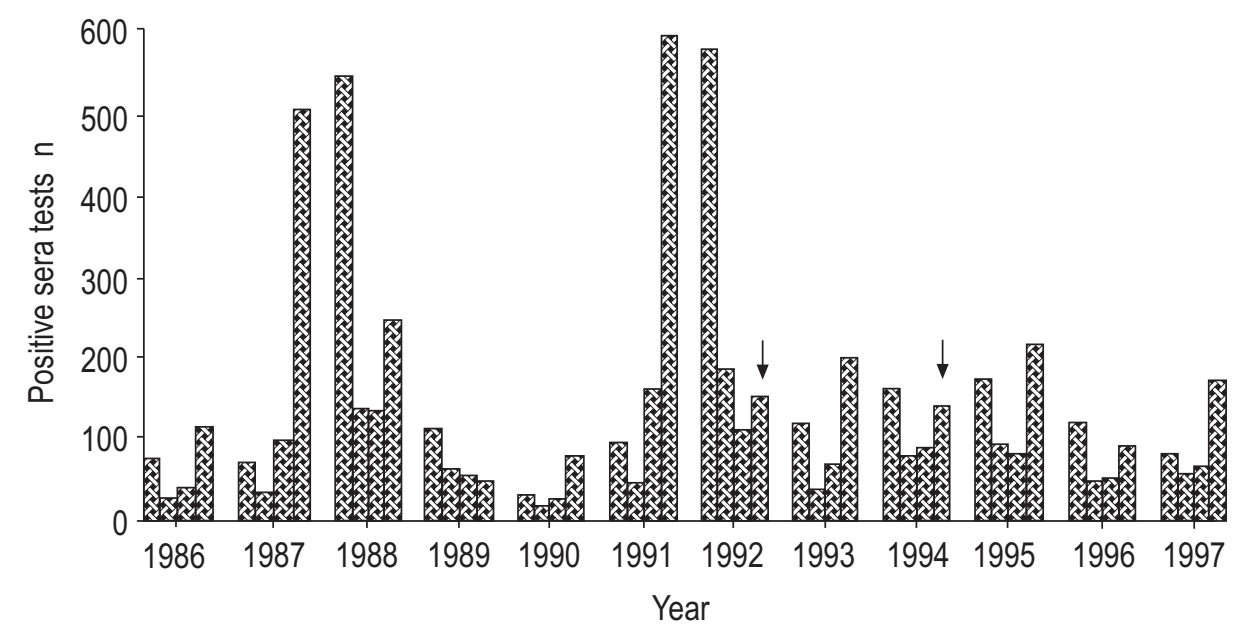

Fig. 1. - Quarterly number of cold agglutinin and anti-Mycoplasma pneumoniae complement fixation test positive sera received at Statens Serum Institut in the period 1986-1997. These data represent serum specimens from most parts of Denmark since testing has been centralized. In each year the four bars represent the four quarters of the year. Arrows indicate the occurence of the two cases of M. pneumoniae. 
Table 1. - The micro-organisms detected in 103 bronchoalveolar lavage fluids from 83 hospitalized human immunodeficiency virus-infected patients

\begin{tabular}{|c|c|c|}
\hline Micro-organisms & $\begin{array}{l}\text { Number } \\
\text { of cases } \\
\text { detected }\end{array}$ & $\begin{array}{c}\text { Detected } \\
\text { as single } \\
\text { agent }\end{array}$ \\
\hline Mycoplasma pneumoniae* & 2 & 0 \\
\hline Legionella pneumophila* & 1 & 0 \\
\hline Chlamydia spp. & 0 & 0 \\
\hline Streptococcus pneumoniae & 13 & 9 \\
\hline Haemophilus influenzae & 10 & 3 \\
\hline Staphylococcus aureus & 4 & 0 \\
\hline Klebsiella pneumonia & 2 & 1 \\
\hline Mycobacterium tuberculosis & 2 & 1 \\
\hline Moraxella catarrhalis & 1 & 1 \\
\hline Pseudomonas spp. & 4 & 1 \\
\hline Streptococcus spp. (haemolytic) & 2 & 0 \\
\hline Streptococcus spp. (nonhaemolytic) & 20 & 7 \\
\hline Corynebacterium spp. & 3 & 0 \\
\hline Staphylococcus albus & 4 & 0 \\
\hline Neisseria catarrhalis & 2 & 0 \\
\hline Enterococcal species & 1 & 0 \\
\hline Serratia marcesens & 1 & 1 \\
\hline Pneumocystis carinii & 23 & 15 \\
\hline Fungi & $11^{+}$ & $2^{*}$ \\
\hline Total & 106 & 41 \\
\hline
\end{tabular}

*: detected by the inhibitor controlled polymerase chain reaction; ${ }^{+}$: Candida albicans (9), mould fungus (1), yeast (1); ${ }^{+}$: one mould fungus, one yeast.

patients. Furthermore, neither $C$. psittasi nor C. trachomatis could be detected in this population. Former studies have shown the prevalence of antibodies to $C$. pneumoniae in HIV-1 infected subjects to be significantly higher than in HIV-negative subjects $[25,26]$, but interpretation of antibody titres in immunocompromised patients may be difficult.

Despite being a well-known intracellular pathogen in immunocompromised patients, L. pneumophila has infrequently been found in HIV-infected patients. BLATT et al. [4] identified eight episodes $(1.7 \%)$ of $L$. pneumophila pneumonia in a population of $401 \mathrm{HIV}$-positive patients. Four of these patients had a coexisting infection with $P$. carinii. This was also demonstrated by BANGSBORG et al. [8], who by direct immunofluorescense found 4 of 161 BAL fluids (2.5\%) from HIV-infected patients to be positive for Legionella spp. In two of these BAL fluids coexisting pathogens were also demonstrated. The findings of the present study are in accordance with the above mentioned studies, as $L$. pneumophila was detected in only one patient $(1 \%)$. The BAL fluid from this patient also contained C. albicans and CMV. The patient was treated with TMP-SMX and ceftazidime, but did not receive any specific legionella treatment as the legionella culture was negative and the PCR investigation of legionella was not performed until after the patient's death. The findings of this study are, however, not in accordance with JIMÉNEZ et al. [12] who found the highest prevalence, detecting L. pneumophila in $8(5 \%)$ of 171 BAL fluids from 151 HIV-positive patients with signs of lower respiratory tract infections by culture and detection with monoclonal antibodies.

M. pneumoniae was only found in the present study in two patients $(2 \%)$, both of whom had symptoms of lower respiratory tract infection. In both cases $M$. pneumoniae was present as a coexisting pathogen. One patient was treated with TMP-SMX and the other with a macrolide. Both patients recovered. As it appears from figure 1, Denmark had an M. pneumoniae epidemic in the last half of 1991, i.e. in the beginning of the period of the study, but both these cases were detected in periods without epidemics and thus, the findings were not expected. WiTT et al. [27] found $M$. pneumoniae in 1 of 21 episodes of communityacquired pneumonia in AIDS patients while OSTERMANN $e t$ $a l$. [13] were unable to detect M. pneumoniae in transbronchial biopsies from $30 \mathrm{HIV}$-positive patients with pneumonia. Among nonimmunocompromised patients the highest incidence of $M$. pneumoniae pneumonia is found among schoolchildren [14], but in children with AIDS relatively few cases of $M$. pneumoniae pneumonia have been described [28]. Thus, M. pneumoniae does not seem to play a major role in lower respiratory tract infections in either children or adults with HIV-infection.

The patient group in the present study was profoundly immunosuppressed with a median CD4 count below $50 \times$ $10^{6} \cdot \mathrm{L}^{-1}$ and the range of pathogens detected in the BAL fluids were typical of those causing lower respiratory tract disease in advanced HIV infection. Coexisting pathogens were found in $22 \%$ of the BAL fluids and, in accordance with a study by Mundy et al. [29], no aetiological agent was identified in $34 \%$ of the BAL fluids. The inability to detect organisms in spite of strong evidence of infection may be due to antibiotic treatment prior to and during the BAL procedure, it may be due to viral infections, as such infections are not uncommon in immunocompromised patients [30], or it may be due to insensitive methods in the detection of the agents. In the immunocompromised patients this may be particularly crucial, as atypical agents such as Legionella spp., C. pneumoniae and M. pneumoniae are commonly diagnosed by serological methods which are less reliable in this patient population. In these cases a rapid diagnosis can be obtained by means of PCR.

In conclusion, pneumonia due to Chlamydia spp., Legionella spp. or Mycoplasma pneumoniae seems to be rare in Danish human immunodeficiency virus-infected patients, but might be considered as a possible cause in cases of treatment failure.

Acknowledgements. The authors are indebted to J. Guldberg, The Research Laboratory, Department of Infectious Diseases, Marselisborg Hospital, University of Aarhus and Birthe Dohn, The Mycoplasma Laboratory, Statens Serum Institut, Copenhagen, for excellent technical assistance, and to L. Jensen, Department of Infectious Diseases, Marselisborg Hospital, for her secretarial assistance.

\section{References}

1. Murray JF, Mills J. Pulmonary infectious complications of human immunodeficiency virus infection. Am Rev Respir Dis 1990; 141: 1356-1372.

2. Nybo Jensen B, Gerstoft J, Højlyng N, et al. Pulmonary pathogens in HIV-infected patients. Scand J Infect Dis 1990; 22: 413-420.

3. Østergaard L, Tarp B, Nybo Jensen B, et al. Semi-nested polymerase for detection of Pneumocystis carinii: implications for diagnosis, prevalence and predictive parameters. Immun Infect Dis 1995; 5: 59-66. 
4. Blatt SP, Dolan MJ, Hendrix CW, Melcher GP. Legionnaires' disease in human immunodeficiency virus-infected patients: eight cases and review. Clin Infect Dis 1994; 18: 227-232.

5. Østergaard L, Huniche B, Andersen PL. Relative bradycardia in infectious diseases. J Infection 1996; 33: 185191.

6. Edelstein PH. Laboratory diagnosis of infections caused by Legionellae. Eur J Clin Microbiol 1987; 6: 4-10.

7. Vickers RM, Stout JE, Yu VL, Rihs JD. Manual of culture methodology for legionella. Semin Respir Infect 1987; 2: 274-279.

8. Bangsborg JM, Nybo Jensen B, Friis-Møller A, Bruun B. Legionellosis in patients with HIV infection. Infection 1990; 18: 342-346.

9. Clark R, Mushatt D, Fazal B. Case report: Chlamydia pneumoniae pneumonia in an HIV-infected man. Am Med Sci 1991; 302: 155-156.

10. Gaydos CA, Fowler CL, Gill VJ, Eiden JJ, Quinn TC. Detection of Chlamydia pneumoniae by polymerase chain reaction-enzyme immunoassay in an immunocompromised population. Clin Infect Dis 1993; 17: 718-723.

11. Augenbraun MH, Roblin PM, Chirgwin K, Landman D, Hammerschlag MR. Isolation of Chlamydia pneumoniae from the lungs of patients infected with the human immunodeficiency virus. JClin Microbiol 1991;29:401-402.

12. Jiménez ML, Aspa J, Padilla B, et al. Fiberoptic bronchoscopic diagnosis of pulmonary disease in $151 \mathrm{HIV-infec-}$ ted patients with pneumonitis. Eur J Clin Microbiol Infect Dis 1991; 10: 491-496.

13. Ostermann A, Klueppelberg U, Wassermann K, Krueger GRF. Non-specific interstitial pneumonia (NIP): immunohistologic screening of etiologlc agents. In vivo 1994; 8: 613-619.

14. Foy HM. Infections caused by Mycoplasma pneumoniae and possible carrier state in different populations of patients. Clin Infect Dis 1993; 17: Suppl. 1, 37-46.

15. Walsh PS, Metzger DA, Higuchi R. Chelex 100 as a medium for simple extraction of DNA for PCR-based typing from forensic material. Biotechniques 1991; 10: 506-513.

16. Chou Q, Russell M, Birch DE, Raymond J, Bloch W. Prevention of pre-PCR mis-priming and primer dimerization improves low-copy-number amplifications. Nucleic Acids Res 1992; 20: 1717-1723.

17. Jensen JS, Ørsum R, Dohn B, Uldum S, Worm AM, Lind K. Mycoplasma genitalium: a cause of male urethritis? Genitourin Med 1993; 69: 265-269.
18. Prince AM, Andrus L. PCR - how to kill unwanted DNA. Biotechniques 1992; 12: 358-360.

19. Pollard DR, Tyler SD, Ng C, Rozee KR. A polymerase chain reaction (PCR) protocol for the specific detection of Chlamydia spp. Mol Cell Probes 1989; 3: 383-389.

20. Don RH, Cox PT, Wainwright BJ, Baker K, Mattick JS. "Touchdown" PCR to circumvent spurious priming during gene amplification. Nucleic Acids Res 1991; 19: 4008 .

21. Storgaard M, Østergaard L, Jensen JS, et al. Chlamydia pneumoniae in otitis media. Clin Infect Dis 1997; 25: 1090-1093.

22. Østergaard L, Møller JK, Birkelund S, Christiansen G, Andersen PL. Development of a nested PCR using timeresolved fluorometri for automated detection of Chlamydia trachomatis. Immun Infect Dis 1994; 4: 36-40.

23. Jensen JS, Søndergård-Andersen J, Uldum SA, Lind $\mathrm{K}$. Detection of Mycoplasma pneumoniae in simulated clinical samples by polymerase chain reaction. APMIS 1989; 97: 1046-1048.

24. Yamamoto H, Hashimoto Y, Ezaki T. Comparison of detection methods for Legionella species in environmental water by colony isolation, fluorescent antibody staining, and polymerase chain reaction. Microbiol Immunol 1993; 37: 617-622.

25. Blasi F, Boschini A, Cosentini R, et al. Outbreak of Chlamydia pneumoniae infection in former injectiondrug users. Chest 1994; 105: 812-815.

26. Monno R, Leone E, Maggi P, Buccoliero G, Valenza MA, Angarano G. Chlamydia pneumoniae: a new opportunistic infectious agent in AIDS? Clin Microbiol Infect 1997; 3: $187-191$.

27. Witt DJ, Craven DE, McCabe WR. Bacterial infections in adult patients with the acquired immune deficiency syndrome (AIDS) and AIDS-related complex. Am J Med 1987; 82: 900-906.

28. Jensen JS, Heilmann C, Valerius NH. Mycoplasma pneumoniae infection in a child with AIDS. Clin Infect Dis 1994; 19: 207.

29. Mundy LM, Auwaerter PG, Oldach D, et al. Communityacquired pneumonia: impact of immune status. Am J Respir Crit Care Med 1995; 152: 1309-1315.

30. Miller RF, Loveday C, Holton J, Sharvell Y, Patel G, Brink NS. Community-based respiratory viral infections in HIV positive patients with lower respiratory tract disease: a prospective bronchoscopic study. Genitourin Med 1996; 72: 9-11. 